\title{
L-Theanine Prevents Long-Term Affective and Cognitive Side Effects of Adolescent $\Delta$-9-Tetrahydrocannabinol Exposure and Blocks Associated Molecular and Neuronal Abnormalities in the Mesocorticolimbic Circuitry
}

\author{
Marta De Felice, ${ }^{1,2}$ Justine Renard, ${ }^{1,2}{ }^{-}$Roger Hudson, ${ }^{1,2}{ }^{-}$Hanna J. Szkudlarek, ${ }^{1,2}$ Brian J. Pereira, ${ }^{1,2}$ \\ ${ }^{1}$ Susanne Schmid, ${ }^{2}$ Walter J. Rushlow, ${ }^{1,2,3}$ and Steven R. Laviolette ${ }^{1,2,3}$ \\ ${ }^{1}$ Addiction Research Group, Schulich School of Medicine \& Dentistry, University of Western Ontario, London, Ontario N6A 5C1, Canada, ${ }^{2}$ Department of \\ Anatomy \& Cell Biology, Schulich School of Medicine \& Dentistry, University of Western Ontario, London, Ontario N6A 3K7, Canada, and \\ ${ }^{3}$ Department of Psychiatry, Schulich School of Medicine \& Dentistry, University of Western Ontario, London, Ontario N6A 3K7, Canada
}

Chronic adolescent exposure to $\Delta$-9-tetrahydrocannabinol (THC) is linked to elevated neuropsychiatric risk and induces neuronal, molecular and behavioral abnormalities resembling neuropsychiatric endophenotypes. Previous evidence has revealed that the mesocorticolimbic circuitry, including the prefrontal cortex (PFC) and mesolimbic dopamine (DA) pathway are particularly susceptible to THC-induced pathologic alterations, including dysregulation of DAergic activity states, loss of PFC GABAergic inhibitory control and affective and cognitive abnormalities. There are currently limited pharmacological intervention strategies capable of preventing THC-induced neuropathological adaptations. L-Theanine is an amino acid analog of L-glutamate and L-glutamine derived from various plant sources, including green tea leaves. L-Theanine has previously been shown to modulate levels of GABA, DA, and glutamate in various neural regions and to possess neuroprotective properties. Using a preclinical model of adolescent THC exposure in male rats, we report that $\mathrm{L}$-theanine pretreatment before adolescent THC exposure is capable of preventing long-term, THC-induced dysregulation of both PFC and VTA DAergic activity states, a neuroprotective effect that persists into adulthood. In addition, pretreatment with L-theanine blocked THC-induced downregulation of local GSK-3 (glycogen synthase kinase 3) and Akt signaling pathways directly in the PFC, two biomarkers previously associated with cannabis-related psychiatric risk and subcortical DAergic dysregulation. Finally, L-theanine powerfully blocked the development of both affective and cognitive abnormalities commonly associated with adolescent THC exposure, further demonstrating functional and long-term neuroprotective effects of $\mathrm{L}$-theanine in the mesocorticolimbic system.

Key words: adolescence; cognition; dopamine; L-theanine; mesocorticolimbic; THC

Significance Statement

With the increasing trend of cannabis legalization and consumption during adolescence, it is essential to expand knowledge on the potential effects of adolescent cannabis exposure on brain development and identify potential pharmacological strategies to minimize $\Delta$-9-tetrahydrocannabinol (THC)-induced neuropathology. Previous evidence demonstrates that adolescent THC exposure induces long-lasting affective and cognitive abnormalities, mesocorticolimbic dysregulation, and schizophrenia-like molecular biomarkers that persist into adulthood. We demonstrate for the first time that L-theanine, an amino acid analog of L-glutamate and L-glutamine, is capable of preventing long-term THC side effects. L-Theanine prevented the development of THC-induced behavioral aberrations, blocked cortical downregulation of local GSK-3 (glycogen synthase kinase 3) and Akt signaling pathways, and normalized dysregulation of both PFC and VTA DAergic activity, demonstrating powerful and functional neuroprotective effects against THC-induced developmental neuropathology.

Received May 2, 2020; revised Sep. 15, 2020; accepted Sep. 22, 2020.

Author contributions: M.D.F. and S.R.L. designed research; M.D.F., J.R., R.H., H.J.S., B.J.P., and W.J.R. performed research; M.D.F., R.H., H.J.S., S.S., and W.J.R. analyzed data; M.D.F. and S.R.L. wrote the paper.
This work was supported by CanaQuest Medical Corp and MITACS Canada.

M.D.F. and S.R.L. declare that the results of this study have been included in intellectual property: U.S. Patent 62/730,253, "L-theanine and tetrahydrocannabinol formulation" (Provisional filled September 12, 2018). The authors declare no other competing financial interests. 


\section{Introduction}

Previous evidence has shown that adolescent $\Delta$-9-tetrahydrocannabinol (THC) exposure increases long-term vulnerability to various neuropsychiatric disorders, including schizophrenia, anxiety, and cognitive impairments (Andréasson et al., 1987; Arseneault et al., 2002; Weiser et al., 2002; Zammit et al., 2002; Fergusson et al., 2003; Stefanis et al., 2004; Ferdinand et al., 2005; Malone et al., 2010; Murray et al., 2017; Krebs et al., 2019). With increasing trends toward cannabis legalization and adolescent consumption, it is critical to characterize the effects of adolescent THC exposure on the developing brain. Moreover, there is an urgent need to identify potential compounds capable of preventing the long-term effects of THC exposure on adolescent brain development.

Given that $\mathrm{THC}$ is a partial $\mathrm{CB} 1$ receptor $(\mathrm{CB} 1 \mathrm{R})$ agonist and $\mathrm{CB1R}$ is enriched in the mesocorticolimbic system (Herkenham et al., 1990), these neural circuits are particularly vulnerable to THC during adolescent neurodevelopment. Translational research has revealed that THC induces long-lasting dysregulation of PFC neuronal activity and oscillatory states resembling schizophrenia-like endophenotypes. These effects include loss of GABAergic inhibitory control of pyramidal neuron activity and hyperactive DAergic activity persisting into adulthood (Renard et al.2017a,b, 2018). These disturbances are accompanied by profound cortical molecular adaptations, resembling phenotypes observed in schizophrenia and mood disorders, such as the loss of glycogen synthase kinase $3 \alpha / \beta$ (GSK-3 $\alpha / \beta$ ), Akt, p70S6K, mammalian target of rapamycin, and GAD67, all critical biomarkers for increased neuropsychiatric risk (Alimohamad et al., 2005; Gururajan and Van Den Buuse, 2014; Renard et al., 2017a,b).

Translational animal models demonstrate a range of behavioral abnormalities following adolescent THC exposure, resulting in sensory filtering deficits (Schneider and Koch, 2003; Wegener and Koch, 2009; Rubino and Parolaro, 2016; Renard et al., 2017a), working memory impairments (Schneider and Koch, 2003; O'Shea et al., 2006; Rubino et al., 2009; Renard et al., 2013; Zamberletti et al., 2014), and social interaction and cognitive disturbances (O'Shea et al., 2006; Renard et al., 2017a). Furthermore, THC exposure is linked to affective dysregulation including heightened anxiety (Llorente-berzal et al., 2013; Renard et al., 2017a) and the development of anhedonialike behaviors (Rubino et al., 2008; Bambico et al., 2010). Despite clinical and preclinical characterization of these phenotypes following adolescent THC exposure, there are limited adjunct pharmacological interventions that can mitigate or prevent these potential neuropathological and/or psychiatric side effects (Murphy et al., 2017; Segal-Gavish et al., 2017; Cuccurazzu et al., 2018).

L-Theanine (thea), an amino acid analog of L-glutamate and L-glutamine, possesses significant neuroprotective properties (Kakuda, 2011; Zukhurova et al., 2013; Chen et al., 2018). For example, L-theanine has been found to have ameliorative effects on positive and anxiety-related symptoms in schizophrenia (Ritsner et al., 2011; Wakabayashi et al., 2012; Ota et al., 2015). A significant improvement in positive and negative syndrome scale (PANSS) scores were observed in schizophrenia patients receiving adjunct L-theanine treatment with antipsychotic medications (Lardner, 2014). While the neurobiological mechanisms underlying

Correspondence should be addressed to Steven R. Laviolette at steven.laviolette@schulich.uwo.ca. https://doi.org/10.1523/JNEUROSCI.1050-20.2020

Copyright $\odot 2021$ the authors the neuroprotective effects of L-theanine have not been clearly elucidated, it has been shown to normalize dopamine (DA), serotonin, and GABA signaling disturbances (Nathan et al., 2006; Lardner, 2014). In addition, because of its structural similarity to glutamate, it has been proposed to produce its therapeutic effects via modulation of glutamatergic dysfunction, which is critically involved in the pathophysiology of schizophrenia (Ota et al., 2015). Indeed, L-theanine has affinities for AMPA, NMDA, and kainate receptors (Kakuda et al., 2002) and has been shown to have long-term inhibitory effects on glutamate release (Kakuda et al., 2008).

In the present study, we hypothesized that L-theanine administration would mitigate the pathologic effects of THC exposure during adolescent neurodevelopment by preventing a battery of neuronal, molecular, and behavioral pathophysiological sequelae linked to THC-related neuropsychiatric side effects. Using an established rodent model of adolescent THC exposure and a combination of behavioral pharmacology, in vivo electrophysiology, and localized molecular pathway analyses in the mesocorticolimbic circuitry, we report for the first time that L-theanine administration powerfully mitigates the long-term negative effects of THC exposure during vulnerable periods of adolescent brain development at the molecular, neuronal, and behavioral levels of pathology.

\section{Materials and Methods}

Animals and housing

Male Sprague Dawley rats were obtained at postnatal day 28 (P28) from Charles River Laboratories. Rats were pair housed in controlled conditions (constant temperature and humidity, $12 \mathrm{~h}$ light/dark cycle) with free access to food and water. All procedures and protocols were approved by appropriate governmental and institutional guidelines.

\section{Drugs}

L-Theanine (Cayman Chemical) was diluted in physiological saline. THC (Cayman Chemical) was dissolved in an ethanol, cremophor, and saline (1:1:18) dilution. Ethanol was evaporated using nitrogen gas to remove it from the final THC solution.

Adolescent THC exposure protocol

Our adolescent THC exposure protocol has been previously described (Renard et al., 2017a). Rats were treated twice daily from P35 to P45 with L-theanine $(10 \mathrm{mg} / \mathrm{kg}$, i.p.) or saline, $10 \mathrm{~min}$ prior to intraperitoneal injections of escalating doses of THC $(2.5 \mathrm{mg} / \mathrm{kg}$, days $1-3 ; 5 \mathrm{mg} / \mathrm{kg}$, days $4-7 ; 10 \mathrm{mg} / \mathrm{kg}$, days $8-11$ ) or vehicle $(\mathrm{VEH})$. The protocol was adapted from the previous rodent studies to overcome CB1 receptor desensitization at chronic exposure phase and that was shown to produce profound and enduring neuropsychiatric phenotypes selectively during adolescent neurodevelopment, but not during adulthood (Renard et al., 2017a). The THC dosing range is designed to mimic the effects of a moderate to heavy use regimen of marijuana on a human adolescent (Renard et al., 2017a,b). All experimental procedures started after a $30 \mathrm{~d}$ drug-free washout period (P75).

\section{Behavioral assays}

Social motivation and cognition. Social interaction testing was performed as described previously (Renard et al., 2017a). Twenty-four hours before testing rats were habituated to a test arena for $13 \mathrm{~min}$ ( $5 \mathrm{~min}$ in the center plus $8 \mathrm{~min}$ in the entire apparatus). The following day, rats were acclimated for $5 \mathrm{~min}$ and then tested for 2 consecutive 8 min phases. In phase 1 , two wire cages, one empty and one containing an unfamiliar male rat, were placed in the apparatus. We measured the propensity of the rat to explore the stranger versus the empty cage and calculated a social motivation index for each test rat (i.e., time spent with the stranger/total time exploring both rats $\times 100$ ). In phase 2 , a new unfamiliar rat was placed in the previously empty cage and the tested rat had a choice between the previously encountered rat versus the novel one. The time spent exploring rats in both chambers was analyzed, and a 
social memory index was calculated for each test rat (i.e., time spent with the novel rat/total time exploring both rats, $\times 100$ ). Locations of stranger and novel rats were randomly counterbalanced between trials. After each test, chambers were cleaned with ethanol to avoid olfactory cue bias. The test was video recorded and analyzed offline with a videotracking system (ANY-maze, Stoelting).

Light-dark box test. The light-dark box test measures anxiety level, based on the innate aversion of rats to bright environments, as previously described (Renard et al., 2017a). The test apparatus consisted of two separated $50 \times 25 \times 37 \mathrm{~cm}$ compartments, connected by a $10-$ $\times 10 \mathrm{~cm}$ opening. One compartment was black and covered with a black lid (the dark box), while the other compartment was white with an open top and brightly illuminated by a lamp located $120 \mathrm{~cm}$ above the apparatus floor, providing 1500 lux at floor level. The test rat was allowed to freely explore both compartments for a period of $8 \mathrm{~min}$. We analyzed times spent in the dark versus the light box measuring total times in either environment, latency to entry for each environment and number of transitions between environments. The test was video recorded and analyzed offline with a video-tracking system (ANY-maze, Stoelting).

Prepulse inhibition of startle reflex. Startle and prepulse inhibition (PPI) testing was performed as previously described (Renard et al., 2017a). Rats were placed in a startle chamber (Med Associates) for a $5 \mathrm{~min}$ acclimatization period over $3 \mathrm{~d}$. On the last day of acclimatization, rats were tested in an input/output function consisting of 12 increasing startle pulses (from 65 to $120 \mathrm{~dB}, 5 \mathrm{~dB}$ increments) to determine the appropriate gain setting for each individual rat. The testing paradigm consisted of the following three phases: acclimatization, habituation (block 1), and PPI measurement (block 2). During acclimatization, rats were exposed to the chambers and white background noise $(68 \mathrm{~dB})$ for $5 \mathrm{~min}$. During block 1, 10 pulse-alone trials $(110 \mathrm{~dB}$ white noise, $20 \mathrm{~ms}$ duration) were delivered at 15-20 s intervals. Block 2 consisted of 9 trials presented 10 times in a pseudorandomized order, and at $30 \mathrm{~s}$ intervals, as follows: 10 pulse-alone trials and 10 of each of three different prepulse-pulse trial types $(72,76,80)$ with interstimulus intervals of $100 \mathrm{~ms}$. The PPI was calculated for each animal and each trial condition as PPI $(\%)=(1-$ average startle amplitude to pulse with prepulse/average startle amplitude to pulse only) $\times 100$.

Object recognition test. Rats were tested using the object recognition task as described previously (Renard et al., 2017b). The test sessions consisted of two $3 \mathrm{~min}$ trials with an intersession interval of $60 \mathrm{~min}$. During the acquisition phase (trial 1), each rat was allowed to explore two identical objects placed $15 \mathrm{~cm}$ from the side wall. For the second trial, one of the objects was replaced with a novel one. The two objects and their locations were randomized and counterbalanced. Object exploration was considered when the rat was sniffing the object. The test was video recorded and analyzed with a video-tracking system (ANYmaze, Stoelting). Exploration times were recorded and used to calculate object recognition index (time spent with novel object/total time exploring both objects) $* 100$.

Sucrose preference test. The sucrose preference test is used to investigate anhedonia (i.e., the inability to experience pleasure). Rats were given access in their home cage to two bottles, containing a $2 \%$ sucrose solution or plain water, respectively, for a period of $4 \mathrm{~d}$. The animals were not isolated, and the test was conducted in their home cages. No food and/or water restriction had been applied before the test, and the positions of the two bottles were randomized daily to avoid any side bias. Water and sucrose intake were measured daily, and the sucrose preference index was calculated for each rat as the percentage of the volume of sucrose solution intake over the total volume of fluid intake averaged across the 4 testing days and across groups.

In vivo electrophysiology

Extracellular single-unit recordings in the PFC and VTA and local field potential (LFP) signals were conducted as described previously (Renard et al., 2017a,b). At adulthood, THC-treated or vehicle-treated rats were anesthetized with urethane $(1.3 \mathrm{~g} / \mathrm{kg}$, i.p.) and placed in a stereotaxic apparatus (David Kopf Instruments), with body temperature maintained at $37 \pm 1^{\circ} \mathrm{C}$ by a heating pad. The scalp was retracted, and one burr hole was drilled above the targeted areas [PFC: anteroposterior (AP), +2.7 to
$+3.5 \mathrm{~mm}$; lateral (L), \pm 0.8 to $\pm 1 \mathrm{~mm}$ from bregma; VTA: AP, $-5.0-5.2$ $\mathrm{mm}$; L, \pm 0.8 to $\pm 1 \mathrm{~mm}$ from bregma], according to the atlas by Paxinos and Watson (2007). Single-unit activity of putative pyramidal neurons in the PFC [dorsoventral (DV), -2.5 to $-4 \mathrm{~mm}$ from the dural surface] and putative DA cells in the VTA (DV, -7 to $-9 \mathrm{~mm}$ from the dural surface) were recorded extracellularly with glass microelectrodes (average impedance, 6-10 M $\Omega$ ) filled with $0.5 \mathrm{~m}$ sodium acetate solution containing $2 \%$ pontamine sky blue (Sigma-Aldrich).

Population spontaneous activity was determined in four to six predetermined recording tracks separated by $200 \mu \mathrm{m}$, and the activity of each neuron was recorded for $5 \mathrm{~min}$. Putative PFC pyramidal cells were identified according to the following previously established criteria: firing frequency $<10 \mathrm{~Hz}$, waveform shape, and action potential duration $>2.5 \mathrm{~ms}$. Cells exhibiting three consecutive spikes with interspike intervals $<45 \mathrm{~ms}$ were classified as burst-firing cells. Putative VTA DA neurons were identified according to previously established electrophysiological features for in vivo extracellular recordings: action potential width $>2.5 \mathrm{~ms}$, spontaneous firing rate between 2 and $5 \mathrm{~Hz}$, a triphasic waveform consisting of a notch on the rising phase followed by a delayed afterpotential, and a single irregular or burst-firing pattern. Single-unit neuronal activity was filtered (bandpass, $0.3-5 \mathrm{kHz}$ ) and individual action potentials were isolated and amplified (MultiClamp $700 \mathrm{~B}$ amplifier, Molecular Devices), digitized at $25 \mathrm{kHz}$ and recorded using a Digidata 1440A Low-noise Data Acquisition System and pClamp software (Molecular Devices).

LFP signals were analyzed using NeuroExplorer (NexTechnologies). LFP were decimated to $1 \mathrm{kHz}$, and low-pass filtered (IIR Butterworth filter at $170 \mathrm{~Hz}$; filter order set to 3). Subsequently, a spectrogram function was used to calculate the power of oscillations at frequencies between 0 and $100 \mathrm{~Hz}$ (window length, $2 \mathrm{~s}$; shift, $0.5 \mathrm{~s}$ ). One-minute-long recording epochs were used for estimating the average power spectrum distributions. Epochs were selected such as either the desynchronized (relatively small signal amplitude and fast oscillations) or synchronized (relatively large signal amplitude with slow oscillations) cortical state could be easily distinguished. Power values for a given frequency were averaged over the time of the recording epoch and normalized so that the sum of all power spectrum values equals 1 . The total power was calculated by adding all of the power values at frequencies between 0 and 59 and 61 and $100 \mathrm{~Hz}$. Power values at $60 \pm 1 \mathrm{~Hz}$ were excluded from all the calculations. The gamma band was defined as the frequency between 30 and $80 \mathrm{~Hz}$, as a result of the following two subcategories: low gamma band, $30-59 \mathrm{~Hz}$; high gamma band, $61-80 \mathrm{~Hz}$.

To perform histologic analyses, at the end of recording sessions, DC current ( $20 \mathrm{~mA}$ for $15 \mathrm{~min}$ ) was passed through the recording micropipette to mark the recording site with an iontophoretic deposit of pontamine sky blue dye.

\section{Western blots}

The Western blotting procedure was performed as previously described (Renard et al., 2017a,b). At the conclusion of the experiments, rats received an overdose of sodium pentobarbital $(240 \mathrm{mg} / \mathrm{kg}$, i.p.; Euthanyl), and the brains were removed and flash frozen. Bilateral micropunch samples of the PFC were obtained for protein isolation. Primary antibody dilutions were as follows: $\alpha$-tubulin, 1:120,000 (Sigma-Aldrich); phosphorylated (p) GSK-3 $\alpha /$ $\beta$ ser21/9 (1:1000; Cell Signaling Technology); total (t) GSK-3 $\alpha / \beta$ ser21/9 (1:1000; Cell Signaling Technology); p-AKT-Thr308, 1:1000 (Cell Signaling Technology); p-AKT-Ser473, 1:1000 (Cell Signaling Technology); t-AKT, 1:1000 (Cell Signaling Technology). Species-appropriate fluorophore-conjugated secondary antibodies (IRDye 680RD and IRDye 800CW, LI-COR) were used at a dilution of 1:10,000. Membranes were scanned using a LICOR Odyssey Infrared Imaging System, and densitometry measurements were obtained using Image Studio analysis software. Target protein bands were normalized to the intensity of the respective $\alpha$-tubulin.

\section{Statistical analysis}

Averaged data from different experiments are presented as the mean \pm SEM. Statistical analyses were performed using GraphPad Prism and SPSS (IBM). The data were analyzed using two-way ANOVA and twoway ANCOVA, where appropriate. Post hoc analyses were calculated 
using Fisher's least significant difference (LSD) test. The significance level was established at $p<0.05$.

\section{Results}

L-Theanine prevents the development of adolescent THC-induced social interaction and memory deficits in adulthood

First, we examined the neuroprotective potential of L-theanine on aberrant social behavior at adulthood (P75) following adolescent THC exposure. Two-way ANOVA revealed that social motivation (Fig. $1 A$ ) was not affected by either adolescent treatment or interaction between factors, as the animals in all of the experimental groups showed preference toward compartment containing stranger rat versus empty cage $(\mathrm{VEH}, n=8$; THC, $n=11$; theanine + THC, $n=12$; theanine, $n=11$; interaction, $F_{(1,38)}=0.003$; THC, $F_{(1,38)}=2.95$; theanine, $F_{(1,38)}=0.05 ; p>0.05$ for all; Fig. $1 B$ ). On the contrary, there was a significant effect of adolescent drug exposure and interaction between factors on the recall of social memory (phase 2 of the test) at adulthood (interaction: $F_{(1,38)}=11.52, p=$ 0.0016; THC: $F_{(1,38)}=8.86, p=0.0051$; theanine: $F_{(1,38)}=6.77, p=0.0131$; Fig. $\left.1 C\right)$. Post hoc analysis revealed that whereas THCexposed rats spent less time exploring the novel rat versus the previously encountered rat compared with controls $(p<0.001$; Fig. $1 C)$, this effect was fully prevented by coexposure with L-theanine $(p<0.001$; Fig. 1C). Moreover, THC rats showed a significant reduction of the recognition score when compared with theanine group ( $p<0.001$; Fig. $1 C$ ).

\section{L-Theanine prevents the development of long-term adolescent THC-induced memory deficits}

Acute or chronic THC exposure has been linked with significant deficits in short-term memory formation (Quinn et al., 2008). Thus, we next examined the effects of adolescent THC exposure on novel object memory formation (Fig. 1D). Consistent with previous reports (Zamberletti et al., 2014; Renard et al., 2017b), significant effects of adolescent treatment and interaction between factors were observed in short-term memory performance (VEH, $n=10$; THC, $n=11$; thea + THC, $n=11$; theanine, $n=10$; interaction, $F_{(1,38)}=51.79$; THC, $F_{(1,38)}=$ 39.34; theanine, $F_{(1,38)}=18.55 ; p \leq 0.0001$ for all; two-way ANOVA; Fig. 1E). Post hoc comparisons revealed short-term memory deficits in adult rats following THC exposure during adolescence when compared with vehicle $(p<0.001)$ and theanine $(p<0.001)$ groups (Fig. $1 E)$. Coadministration of THC with L-theanine was able to restore the short-term memory deficits observed in THC-exposed rats $(p<0.001$; Fig. $1 E)$.

A
SOCIAL INTERACTION
B
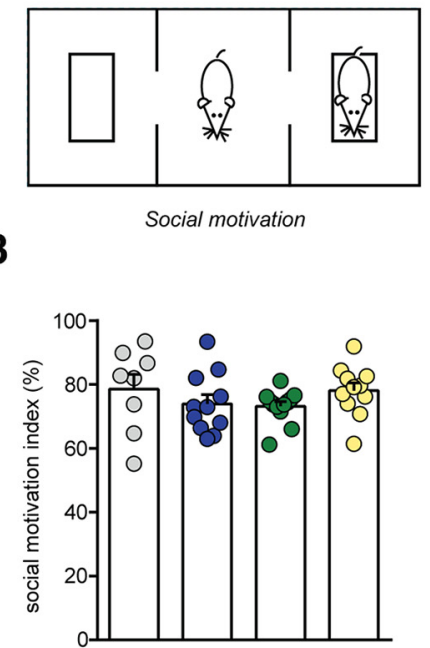

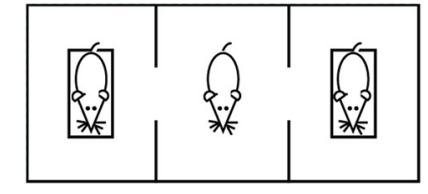

C
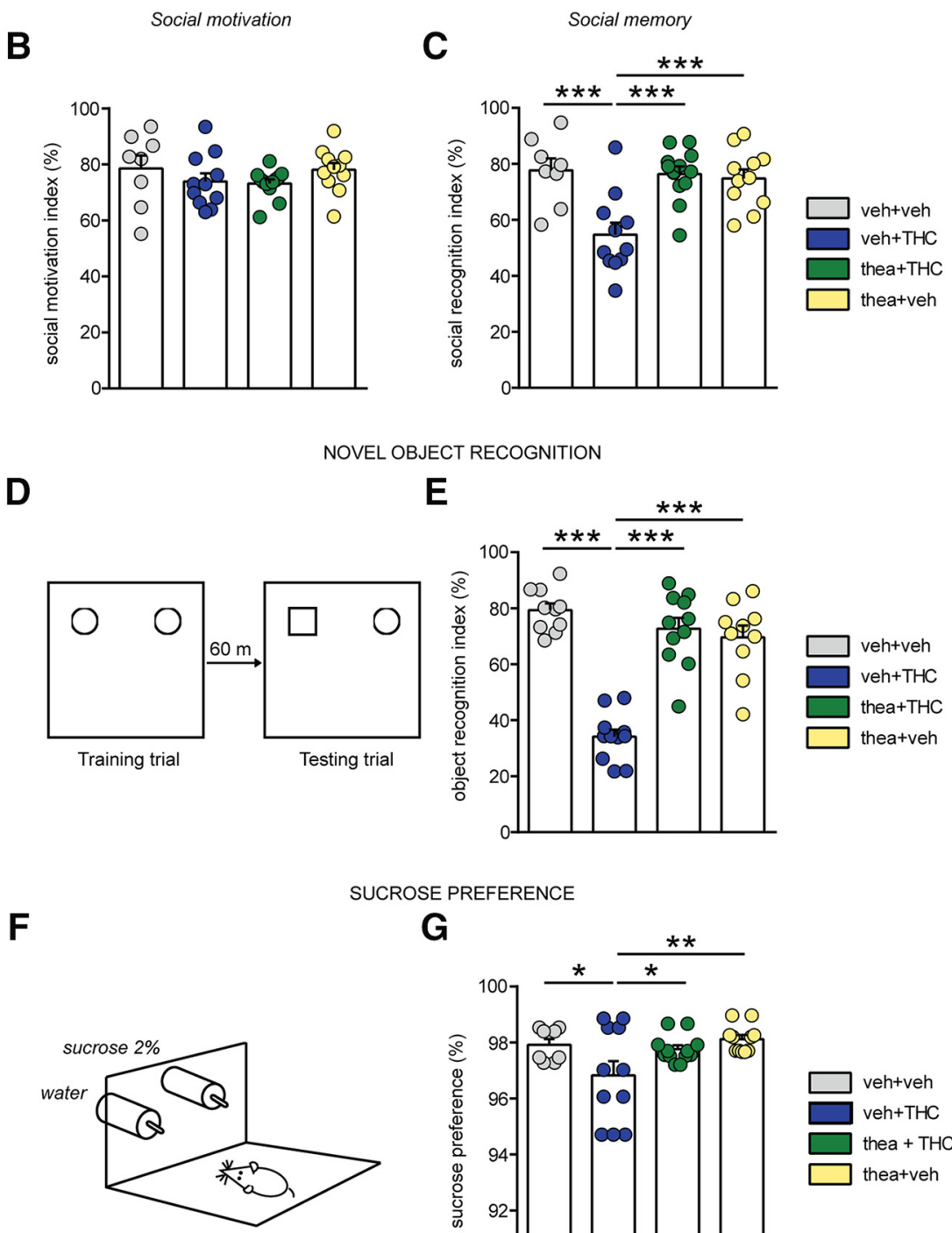

SUCROSE PREFERENCE

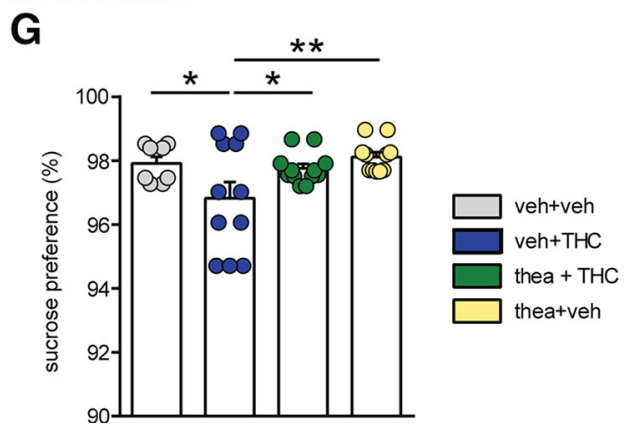

Figure 1. Effects of L-theanine pre-exposure on social, cognitive, and depressive aberrations induced by adolescent THC exposure. $\boldsymbol{A}$, Schematic representation of the social interaction apparatus. $\boldsymbol{B}, \boldsymbol{C}$, Chronic THC exposure did not influence social motivation $(\boldsymbol{B})$ but induced significant deficits in social memory $(\boldsymbol{C})$. L-theanine prevents the THC-induced impairments in social recognition (vehicle, $n=8$; THC, $n=11$; thea + THC, $n=12$; theanine, $n=11$; two-way ANOVA, post hoc Fisher's LSD; ***p $<0.001$ ). $D$, Schematic protocol representation for object memory test. $E$, L-Theanine prevented THCinduced short-term memory impairments (vehicle, $n=10$; THC, $n=11$; thea + THC, $n=11$; theanine, $n=10$; two-way ANOVA, post hoc Fisher's LSD; $* * * p<0.001)$. $\boldsymbol{F}$, Schematic representation of the two-bottle sucrose preference test. G, L-Theanine prevented THC-induced anhedonia effects (vehicle, $n=8$; THC, $n=11$; thea + THC, $n=12$; theanine, $n=11$; two-way ANOVA, post hoc Fisher's LSD; $* p<0.05, * * p<0.01$ ).

L-Theanine prevents the development of adolescent THCinduced anhedonic and anxiety-like behaviors

Another shared endophenotype of schizophrenia and chronic THC exposure is anhedonia, defined as a diminished ability to experience pleasure (Rubino et al., 2008). To examine anhedonic-like behaviors, we used the sucrose preference test (Fig. $1 F)$. Two-way ANOVA revealed a significant main effect of adolescent THC exposure in sucrose preference index (VEH, $n=8$; 
A

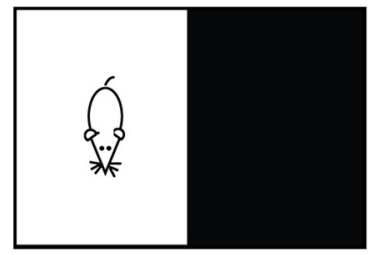

D

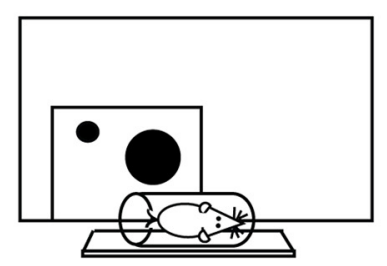

LIGHT/DARK BOX
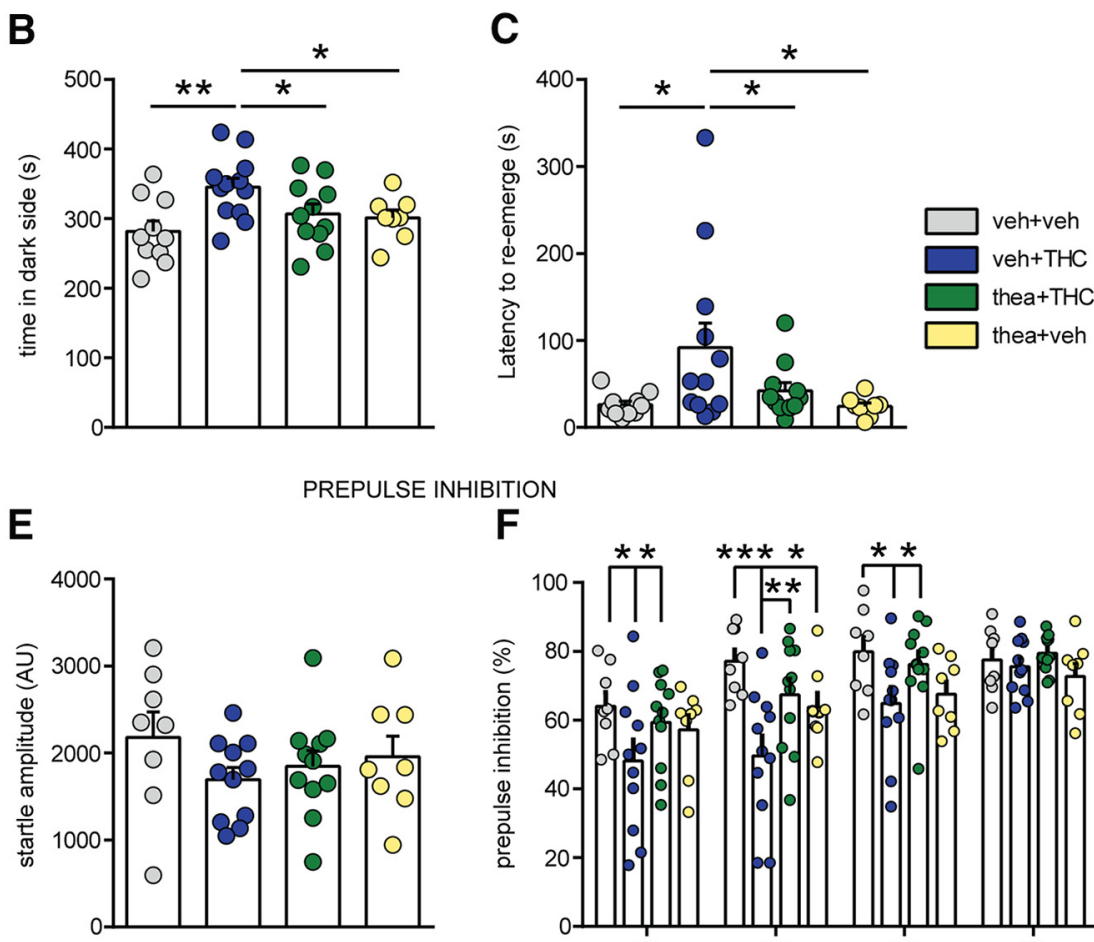

$\mathbf{F}$

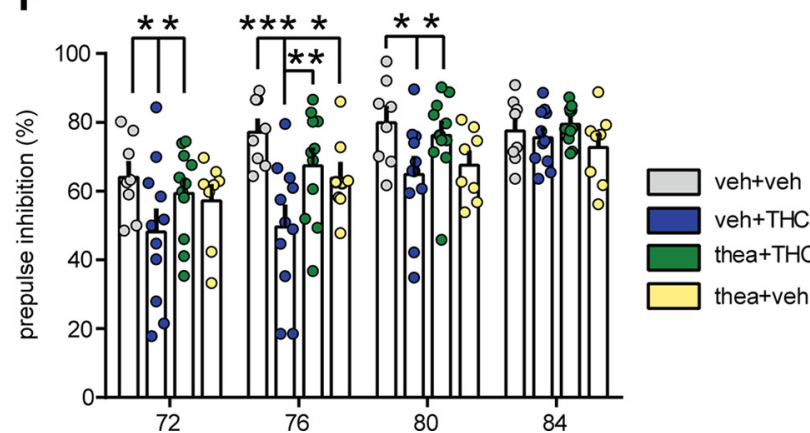

Figure 2. Effects of L-theanine pre-exposure on affective and sensorimotor gating impairments induced by adolescent THC exposure. $A$, Schematic representation of light-dark box anxiety test. $B$, $C$, Adolescent THC-treated rats spent more time in the dark $(\boldsymbol{B})$ and re-emerged later $(\boldsymbol{C})$, indicating increased anxiety levels. L-Theanine administration partially prevents long-term THC-induced anxiety (vehicle, $n=10$; THC, $n=12$; thea + THC, $n=11$; theanine, $n=8$; two-way ANOVA, post hoc Fisher's LSD; $* p<0.05, * * p<0.01)$. $\boldsymbol{D}$, Schematic representation of PPI apparatus setup. $\boldsymbol{E}$, Average startle amplitude response was not affected by any treatment. $\boldsymbol{F}$, Compared with vehicle-treated controls, THC-treated rats exhibited impairments in sensory motor gating, which were fully prevented by L-theanine (vehicle, $n=8$; THC, $n=11$; thea + THC, $n=11$; theanine, $n=8$; two-way repeated-measures ANOVA, post hoc Fisher's LSD; $* p<0.05, * * p<0.01, * * * p<0.001$ ).

THC, $n=11$; thea + THC, $n=12$; theanine, $n=11 ; F_{(1,38)}=5.68$, $p=0.0222$; Fig. $1 G)$. Post hoc analyses revealed that while adolescent THC-exposed rats showed decreased sucrose preference relative to $\mathrm{VEH}$ controls $(p<0.05$; Fig. $1 G)$, this effect was blocked in rats receiving L-theanine pretreatment $(p<0.05$; Fig. $1 G)$. In addition, the THC group exhibited a significant reduction when compared with theanine rats $(p<0.01$; Fig. $1 G)$. While chronic THC exposure has previously been reported to influence feeding behaviors in rats (Rubino et al., 2008), we found that before commencing behavioral testing in adulthood, there were no significant body weight differences between experimental cohorts, suggesting that THC exposure likely had no impact on sucrose preference consumption behaviors (data not shown).

We next evaluated the potential effects of L-theanine on THC-induced anxiogenic behaviors using the light-dark box test (Fig. 2A). A main effect of THC treatment and interaction between factors were observed in time spent in the dark environment (VEH, $n=10$; THC, $n=12$; thea + THC, $n=11$; theanine, $n=8$; interaction: $F_{(1,37)}=4.30, p=0.0452$; THC: $F_{(1,37)}=6.12$, $p=0.0181$; two-way ANOVA; Fig. $2 B$ ), while a significant effect of adolescent THC exposure was found in latency to re-enter into the light environment $\left(F_{(1,37)}=5.50, p=0.0245\right.$; two-way ANOVA; Fig. 2C). Post hoc analyses revealed that THC-treated rats spent more time in the dark side $(p<0.01$; Fig. $2 B)$ and reemerged later $(p<0.05$; Fig. $2 C)$ when compared with VEH controls. Similarly, THC rats exhibited a significant anxiogenic behavior compared with the theanine group in time spent in the dark ( $p<0.05$; Fig. $2 B)$, as well as in second transition from dark to light $(p<0.05$; Fig. $2 C)$. L-Theanine prevented THC-induced anxiogenic effects in terms of times spent in the dark zone and in latency of dark/light environmental transitions $(p<0.05$ for both; Fig. 2B,C).

\section{L-Theanine prevents the development of adolescent THC-} induced sensorimotor gating deficits in adulthood

Core deficits in sensorimotor filtering are found in both schizophrenia and following chronic THC exposure (Braff and Geyer, 1990; Renard et al., 2017a). Thus, we next used a paired-pulse inhibition procedure to measure this endophenotype (Fig. 2D). As shown in Figure $2 E$, baseline startle amplitudes were not affected by adolescent treatment (VEH, $n=8$; THC, $n=11$; thea + THC, $n=11$; theanine, $n=8$; interaction, $F_{(1,34)}=0.81$; THC, $F_{(1,34)}=$ 2.03; theanine, $F_{(1,34)}=0.03 ; p>0.05$ for all; two-way ANOVA). However, two-way ANOVA revealed a significant interaction between treatment and prepulse intensities $\left(F_{(9,102)}=2.41\right.$; $p=0.0160$; Fig. $2 F)$ as well as a main effect of treatment $\left(F_{(3,34)}=\right.$ $3.59 ; p=0.0235)$ and prepulse intensities $\left(F_{(3,102)}=32.92\right.$; $p<0.0001)$. Post hoc comparisons revealed that whereas THC rats showed significant PPI deficits at prepulse intensity levels of $72 \mathrm{~dB}(p<0.05), 76 \mathrm{~dB}(p<0.001)$, and $80 \mathrm{~dB}(p<0.05)$, L-theanine treatment with THC was able to reverse THC-induced impairments at same intensity levels (THC vs thea + THC: $72 \mathrm{~dB}, p<0.05 ; 76 \mathrm{~dB}, p<0.01$; and $80 \mathrm{~dB}, p<0.05$; Fig. $2 F)$. Moreover, THC rats showed a significant impairment at $76 \mathrm{~dB}$, compared with the theanine group $(p<0.05$; Fig. $2 F)$.

\section{L-Theanine treatment normalizes THC-induced} mesocorticolimbic neuronal aberrations

Dysregulation of cortical pyramidal neuron and subcortical DAergic neuronal activities are core endophenotypes associated with both schizophrenia and chronic THC exposure and are 
mechanistically linked to their effects on affective and cognitive abnormalities (Kambeitz et al., 2014; Renard et al., 2017a,b). Given our above-described findings showing that L-theanine + THC treatment effectively blocked THC-induced behavioral abnormalities, we next examined whether L-theanine may similarly block PFC or subcortical VTA DA neuronal dysregulations following adolescent THC exposure.

Since multiple cells were recorded from each individual rat and were considered as independent observations, we assess the variability among subjects including their identity as covariate in two-way ANCOVA. While statistical analysis did not reveal a significant effect of subjects $\left(F_{(1,129)}=1.95, p=0.165\right)$, sampling the population of VTA revealed a main effect of adolescent THC treatment and interaction between fixed factors (cells/rats: $\mathrm{VEH}$, $n=36 / 6$; THC, $n=26 / 5$; thea + THC, $n=41 / 4$; theanine, $n=31 /$ 5; THC: $F_{(1,129)}=5.23, p=0.024$; interaction: $F_{(1,129)}=4.39$, $p=0.038$; two-way ANCOVA; Fig. 3A). Post hoc comparisons showed an increase in DA neuronal frequency in THC-treated rats compared with the vehicle-treated rats $(p<0.01$; Fig. $3 A)$ and theanine by itself $(p<0.05$; Fig. $3 A)$, while coadministration with $\mathrm{L}$-theanine was able to normalize this effect $(p<0.05$; Fig. $3 A$ ). Similarly, two-way ANCOVA revealed no effects of subjects on bursting activity of putative VTA DA neurons $\left(F_{(1,129)}=\right.$ $0.001, p=0.975)$, and a significant interaction between theanine and THC treatments $\left(F_{(1,129)}=14.89, p=0.000\right.$; Fig. $\left.3 B\right)$. Post hoc comparisons demonstrated that THC rats showed an increase in bursting activity when compared with the vehicle group ( $p<0.01$ for both; Fig. $3 B$ ), which was not prevented by L-theanine coexposure ( $p>0.05$; Fig. $3 B)$. As shown in Figure $3 B$, adolescent treatment with L-theanine increased DA burst firing when compared with vehicle-treated rats $(p<0.001$; Fig. $3 B)$ and the theanine + THC combined group $(p<0.01$; Fig. $3 B)$. Figure $3 C$ shows representative traces and rate histograms of VTA DA neurons recorded from each treatment group.

Subsequently, we analyzed the spontaneous activity of putative pyramidal neurons and LFP in PFC. The study of the whole population, including tonic and bursting cells, revealed significant effects of subjects and treatments (cells/rats; VEH, $n=45 / 7$; THC, $n=37 / 5$; thea + THC, $n=74 / 6$; theanine, $n=40 / 5$; rats: $F_{(1,191)}=4.83, p=0.029$; theanine: $F_{(1,191)}=6.09, p=0.014$; THC: $F_{(1,191)}=5.94, p=0.016$; two-way ANCOVA; Fig. $\left.3 D\right)$. However, post hoc comparisons did not reveal any differences between groups.

When a more detailed analysis of bursting in PFC putative pyramidal neurons was performed, a notable effect of THC treatment, but not of the individual subjects, emerged (cells/ rats: VEH, $n=37 / 7$; THC, $n=31 / 5$; thea $+\mathrm{THC}, n=57 / 6$; theanine, $n=20 / 5$; rats: $F_{(1,140)}=0.57, p=0.453$; THC: $F_{(1,140)}=$ $8.08, p=0.005$; two-way ANCOVA; Fig. $3 E$ ). Post hoc comparisons indicated that bursting rate was enhanced in adolescent THC-exposed rat compared with the vehicle and theanine groups ( $p<0.001$ for both; Fig. $3 E$ ), and coadministration with L-theanine fully prevented this hyperactive PFC bursting state $(p<0.001$; Fig. $3 E)$. Traces and rate histograms of representative PFC pyramidal neurons recorded from each group are shown in Figure $3 F$.

Aberrant gamma-band oscillations in the PFC are well established functional correlates of both schizophrenia and chronic neurodevelopmental THC exposure (Williams and Boksa, 2010; Raver et al., 2013). Accordingly, we next analyzed PFC LFP recordings to examine potential effects on gamma-band oscillation patterns. Epochs of the desynchronized [i.e., small-voltage $(<0.5 \mathrm{mV})$ and fast oscillations] state were examined (Fig. $4 A)$.
Two-way ANCOVA did not reveal any effect of subjects $\left(F_{(1,72)}=1.46, p=0.231\right)$. However, there was a significant interaction between THC and theanine treatment in gammaband desynchronized activity, calculated as the sum of the frequency power values between 30 and $80 \mathrm{~Hz}$ (recording sites/ rats: VEH, $n=20 / 6$; THC, $n=17 / 3$; thea + THC, $n=23 / 4$; theanine, $n=17 / 4 ; F_{(1,72)}=8.73, p=0.004$; Fig. $\left.4 C\right)$. Post hoc analysis revealed that, during the desynchronized states, THCtreated rats showed a significant reduction in gamma oscillations when compared with the vehicle counterparts $(p<0.05$; Fig. $4 C$ ), which was fully normalized by L-theanine pre-exposure $(p<0.001$; Fig. $4 C)$. Moreover, coapplication of theanine and THC was yielding significantly higher gamma power scores than theanine alone ( $p<0.05$; Fig. $4 C)$.

\section{L-Theanine prevents the development of maladaptations in schizophrenia-related PFC molecular biomarkers following adolescent THC exposure}

We next examined protein expression levels of select molecular signaling pathways associated with both schizophrenia and neurodevelopmental THC exposure. Previous reports have demonstrated a dysregulation of both the GSK- $3 \alpha / \beta$ and AKT signaling pathways in postmortem cortical tissue samples of schizophrenic subjects (Emamian, 2012) as well as in adult rats following THC adolescent exposure (Renard et al., 2017a), demonstrating their importance as common neuropathological biomarkers. PFC Western blots measuring p-GSK- $3 \alpha$ revealed a significant interaction between adolescent THC and theanine treatment (VEH, $n=8$; THC, $n=7$; thea + THC, $n=8$; theanine, $n=8 ; F_{(1,27)}=$ 8.03, $p=0.0086$; two-way ANOVA; Fig. $5 A$ ). Post hoc comparisons revealed that THC exposure during adolescence induced longterm reduction in p-GSK-3 $\alpha$ expression level ( $p<0.05$; Fig. $5 A)$ and that $\mathrm{L}$-theanine pretreatment reversed this downregulation effect $(p<0.05$; Fig. $5 A$ ). Expression of total GSK-3 $\alpha$ was unaffected ( $n=8$ for each group; interaction, $F_{(1,28)}=0.85$; THC, $F_{(1,28)}$ $=0.10$; theanine, $F_{(1,28)}=0.59 ; p>0.05$ for all; two-way ANOVA; Fig. 5A). A significant interaction between factors was observed in the ratio of p-GSK- $3 \alpha$ /total GSK- $3 \alpha$ expression (VEH, $n=8$; THC, $n=7$; thea + THC, $n=8$; theanine, $n=8 ; F_{(1,27)}=8.54$, $p=0.0069$; two-way ANOVA; Fig. 5A). Post hoc analysis revealed that the ratio of $\mathrm{p}-\mathrm{GSK}-3 \alpha /$ total GSK- $3 \alpha$ expression was reduced following adolescent THC exposure ( $p<0.05$; Fig. $5 A)$ and prevented by the coadministration of L-theanine ( $p<0.05$; Fig. $5 A)$.

Similarly, we found a significant interaction between THC and theanine treatment in $\mathrm{p}-\mathrm{GSK}-3 \beta$ expression level (VEH, $n=8$; THC, $n=7$; thea + THC, $n=8$, theanine, $n=8 ; F_{(1,27)}=$ 12.57, $p=0.0015$; two-way ANOVA; Fig. $5 A$ ). Post hoc analysis revealed that THC administration during adolescence reduced PFC p-GSK- $3 \beta$ expression ( $p<0.05$; Fig. $5 A$ ), whereas L-theanine treatment prevented this effect $(p<0.001$; Fig. $5 A)$. Moreover, PFC p-GSK-3 $\beta$ expression was significantly higher in rats exposed to the combination of theanine and THC, compared with the theanine-alone group ( $p<0.05$; Fig. $5 A$ ). Total GSK-3 $\beta$ PFC expression was not significantly affected by adolescent treatment $\left(n=8\right.$ for each group; interaction, $F_{(1,28)}=$ 0.83 ; THC, $F_{(1,28)}=0.16$; theanine, $F_{(1,28)}=0.49 ; p>0.05$ for all; two-way ANOVA; Fig. 5A). A significant interaction between treatments was observed in the ratio of p-GSK-3 $\beta$ /total GSK- $3 \beta$ expression (VEH, $n=8$; THC, $n=7$; thea + THC, $n=8$; theanine, $n=8 ; F_{(1,27)}=8.04, p=0.0086$; two-way ANOVA; Fig. $\left.5 A\right)$. Post hoc analysis revealed a significant increase following the coexposure of theanine and THC compared with THC-treated $(p<0.01$; Fig. 5A) and theanine-alone ( $p<0.05$; Fig. 5A) groups. Analysis 
VTA DOPAMINE NEURONS

A

Spontaneous firing

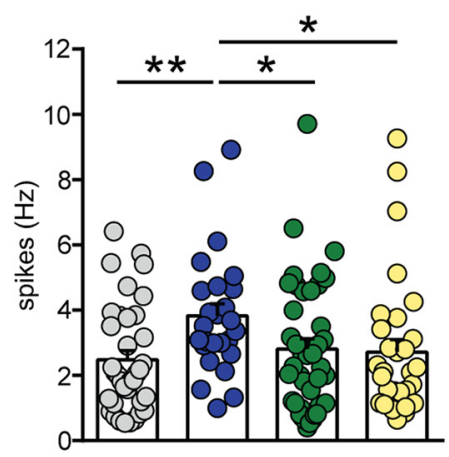

B

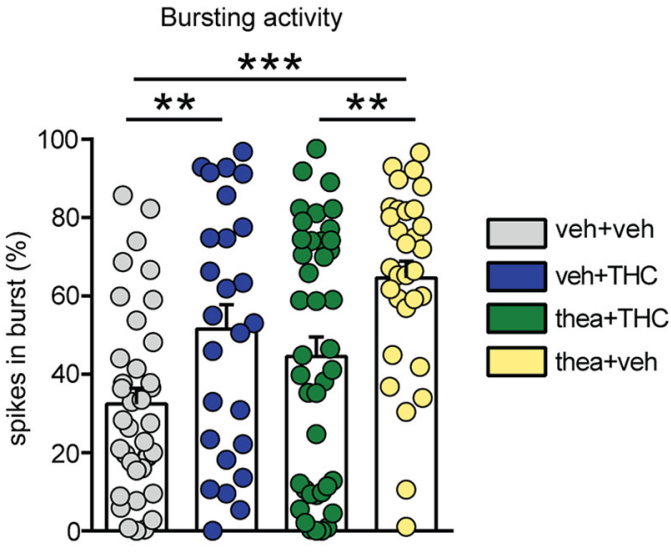

C
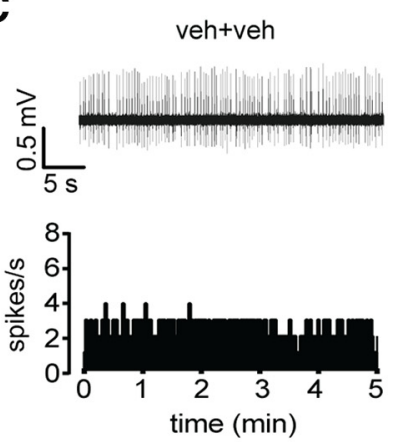

D

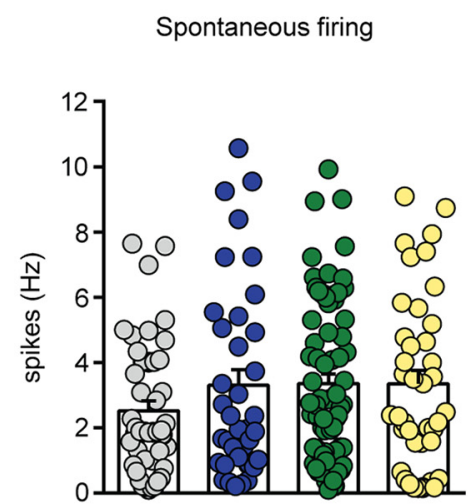

veh $+\mathrm{THC}$
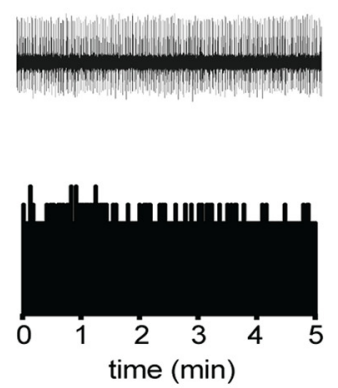
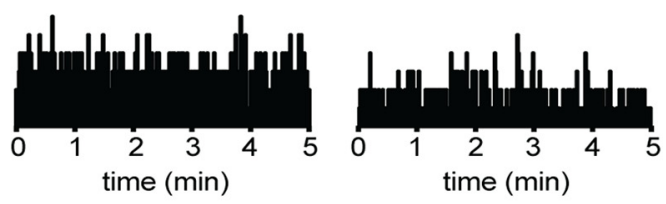

PFC PYRAMIDAL NEURONS

E

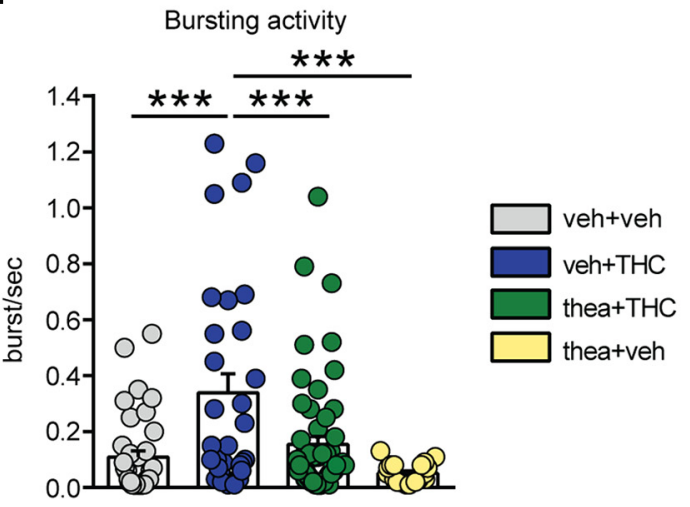

$\mathbf{F}$
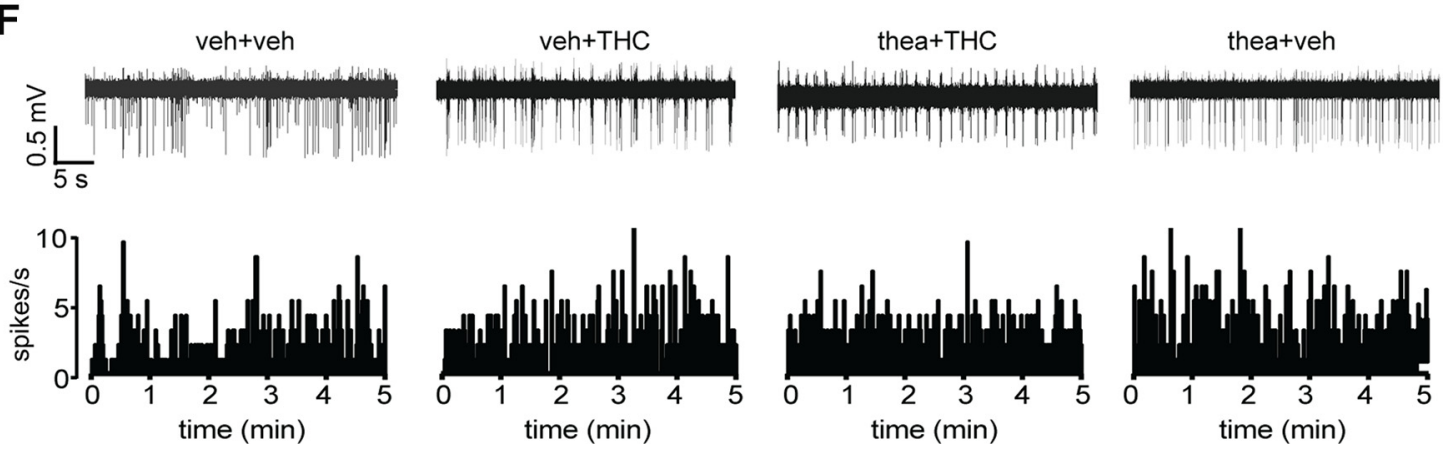

Figure 3. Effects of L-theanine exposure on adolescent THC-induced alterations of VTA DA and PFC pyramidal activity. $A, \mathrm{~L}-$ Theanine prevents the subcortical hyperdopaminergic state induced by THC in terms of firing frequency. $\boldsymbol{B}$, THC-treated rats exhibited an increase in bursting activity, which was not prevented by L-theanine + THC or L-theanine alone. Rats exposed to L-theanine alone showed a higher bursting activity when compared with the other groups ( $n=36$ cells from 6 vehicle rats; $n=26$ cells from 5 THC rats; $n=41$ cells from 4 thea + THC rats; $n=31$ 
of PFC AKT expression patterns revealed a significant interaction between factors in p-AKT-Thr308 (VEH, $n=8$; THC, $n=7$; thea + THC, $n=8$; theanine, $n=8 ; F_{(1,27)}=9.14, p=0.0054$; two-way ANOVA; Fig. 5B). Post hoc comparisons revealed that THC exposure strongly reduced PFC $\mathrm{p}$-AKT-Thr308 expression relative to VEH controls $(p<0.01$; Fig. $5 B)$. Moreover, the theanine group showed a decrease in $\mathrm{p}$-AKT-Thr308 expression levels when compared with controls $(p<0.05$; Fig. $5 B)$. Interaction between theanine and THC was observed in the ratio of p-AKT-Thr308/total AKT-Thr308 expression $\left(F_{(1,27)}=6.87, p=0.0143\right.$; two-way ANOVA; Fig. 5B). Post hoc comparison revealed that the ratio of p-AKT-Thr308/total AKT-Thr308 expression was significantly reduced following THC exposure during adolescence $(p<0.05$; Fig. $5 B$ ). The total Akt protein expression level was not affected by the treatment $\left(n=8\right.$ for each group; interaction; $F_{(1,28)}=0.01$; THC, $F_{(1,28)}=0.21$; theanine, $F_{(1,28)}=0.12 ; p>0.05$ for all; twoway ANOVA; Fig. 5B). Moreover, no difference was observed in p-AKT-Thr473 expression level $(n=8$ for each group; interaction, $F_{(1,28)}=0.28$; THC, $F_{(1,28)}=0.33$; theanine, $F_{(1,28)}=1.30 ; p>0.05$ for all; two-way ANOVA; Fig. $5 B$ ) as well as in the ratio of pAKT-Thr473/total AKT-Thr473 expression (interaction, $F_{(1,28)}=$ 0.60 ; THC, $F_{(1,28)}=0.35$; theanine, $F_{(1,28)}=0.47 ; p>0.05$ for all; two-way ANOVA; Fig. 5B).

\section{Discussion}

Clinical and preclinical evidence has demonstrated that adolescent THC exposure induces a wide range of neuropsychiatric side effects, including psychotomimetic mood/anxiety-related disturbances and cognitive deficits (Rubino et al., 2008; Llorente-berzal et al., 2013; Renard et al., 2013, 2017a; Rubino and Parolaro, 2016). However, there are currently limited adjunct pharmacological treatments capable of preventing these long-term side effects (Murphy et al., 2017; Segal-Gavish et al., 2017; Cuccurazzu et al., 2018). Our findings demonstrate for the first time that the administration of L-theanine, before THC exposure, can powerfully prevent a host of well established neuronal, behavioral, and molecular biomarkers in the mesocorticolimbic circuitry that are associated with THC-induced neuropathophysiology.

In line with previous reports (Renard et al., 2017a,b), adolescent THC exposure induced a range of long-term behavioral symptoms resembling neuropsychiatric phenotypes, including deficits in social recognition and short-term memory, anhedonia, sensorimotor gating impairments, and increased anxiety. Remarkably, L-theanine significantly normalized all these THC-related neurodevelopmental behavioral phenotypes into adulthood. Previously, acute L-theanine exposure has been found to ameliorate select cognitive deficits and to have proattentional effects independently of cannabis-related phenomena. For example, Tamano et al. (2013) found that acute stress-

\footnotetext{
cells from 5 theanine rats; two-way ANCOVA, post hoc Fisher's LSD; $* p<0.05, * * p<0.01$, $* * * p<0.001)$. C, Traces and rate histograms of representative VTA DA neurons recorded from each group. $D$, The firing rate of spontaneous PFC putative pyramidal neurons was not altered by adolescent THC treatment ( $n=45$ cells from 7 vehicle rats; $n=37$ cells from 5 THC rats; $n=74$ cells from 6 thea + THC rats; $n=40$ cells from 5 theanine rats; two-way ANCOVA, post hoc Fisher's LSD; $p>0.05)$. $\boldsymbol{E}$, However, the analysis of bursting activity revealed that L-theanine significantly prevented hyperbursting phenotypes induced by adolescent THC ( $n=37$ cells from 7 vehicle rats; $n=31$ cells from 5 THC rats; $n=57$ cells from 6 thea + THC rats; $n=20$ cells from 5 theanine rats; two-way ANCOVA, post hoc Fisher's LSD; $* * * p<0.001)$. $\boldsymbol{F}$, Traces and rate histograms of representative PFC pyramidal neurons recorded from each group.
}

$\leftarrow$ induced object recognition memory impairments and associated hippocampal synaptic plasticity deficits could be reversed by L-theanine administration in rats. Social interaction memory deficits are cardinal features of schizophrenia-related disorders, and episodic deficits in memories for social interactions are perturbed during all phases of the illness (Lee et al., 2018). Consistent with previous reports (Renard et al., 2017a), we observed significant social memory deficits following adolescent THC exposure. Similar to object recognition deficits, these social cognition impairments were completely reversed by L-theanine administration. While no previous reports have examined the effects of L-theanine specifically on social memory processing, a previous report (Park et al., 2011) demonstrated that L-theanine administration significantly improved semantic and episodic memory indices in patients with mild cognitive impairments, an effect that correlated with increased theta-wave activity in several neural regions, including the frontal cortices.

Consistent with clinical and preclinical findings (Rubino et al., 2008; Bambico et al., 2010; Renard et al., 2017a), we observed long-term anhedonia and increased anxiety-like behaviors following adolescent THC exposure. Remarkably, L-theanine blocked both anhedonic and anxiety-related phenotypes induced by adolescent THC. Previous evidence has suggested that acute L-theanine may possess both mood-enhancing and anxiolytic effects (Ritsner et al., 2011; Yin et al., 2011; Lardner, 2014; Ota et al., 2015). Moreover, antidepressant-like effects of L-theanine have been demonstrated in the forced swim and tail suspension tests in preclinical studies (Yin et al., 2011; Wakabayashi et al., 2012). Preclinical studies have also found that the acute antidepressant and anxiolytic effects of $\mathrm{L}$-theanine may be related to its ability to increase levels of DA and serotonin, directly within the mesocorticolimbic circuitry (Shen et al., 2019). Furthermore, long-term administration of $\mathrm{L}$-theanine has been found to reduce anxiety-related symptoms in schizophrenia, as measured by the Hamilton anxiety rating scale (Ritsner et al., 2011; Lardner, 2014; Ota et al., 2015).

While the precise mechanisms underlying adolescent THCinduced anxiogenic and anhedonic-like effects are not entirely understood, previous evidence has demonstrated that acute activation of CB1Rs in the rat PFC and modulation of PFC neuronal firing and bursting rates can strongly modulate fear-related memory formation and sensitivity to fear-related cues (Laviolette and Grace, 2006; Tan et al., 2011; Draycott et al., 2014). CB1Rmediated potentiation of fear-related memory encoding depends on a pathologic amplification of PFC neuron associative bursting rates (Laviolette and Grace, 2006; Tan et al., 2011). In addition, intra-PFC CB1R activation has been shown to potentiate the anxiogenic properties of associative footshock via hyperactivating subcortical VTA DA neuron firing and bursting rates (Draycott et al., 2014). While the present study used long-term neurodevelopmental protocols for THC and L-theanine exposure, our finding that L-theanine prevented the effects of chronic CB1R stimulation (via adolescent THC exposure) on inducing PFC-related and VTA-related neuronal pathologies may suggest a mechanism by which L-theanine may block longer-term dysregulation of anxiety-related and mood-related phenotypes via normalization of PFC/VTA-related neuronal dysregulation.

The inability to filter out irrelevant sensory stimuli, demonstrated by deficits in sensorimotor gating, represents a crucial endophenotype of schizophrenia (Braff and Geyer, 1990). The PFC, via complex interactions with brainstem circuitry, is a critical component of normal PPI behavioral function (Swerdlow et 
A
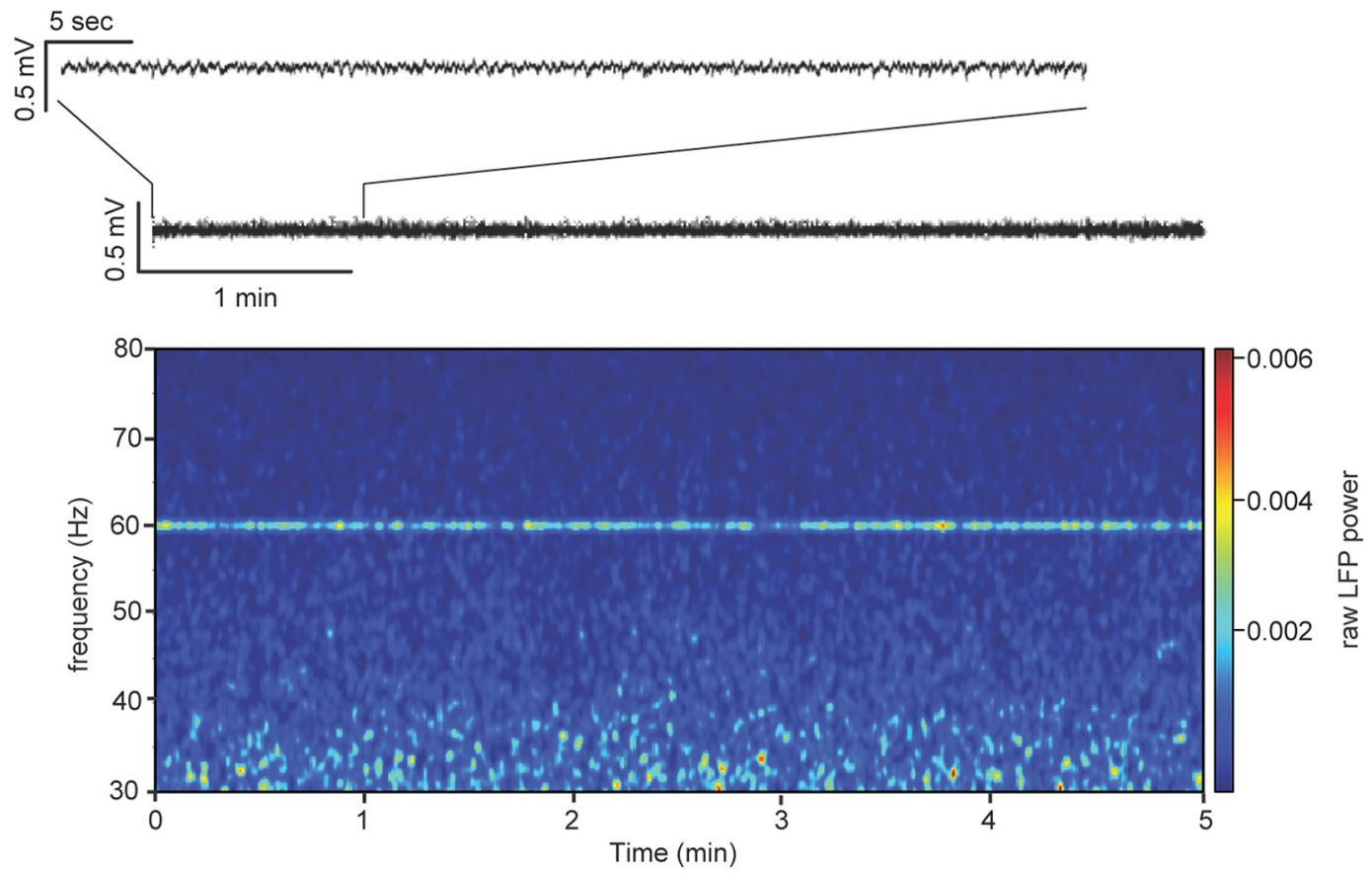

B

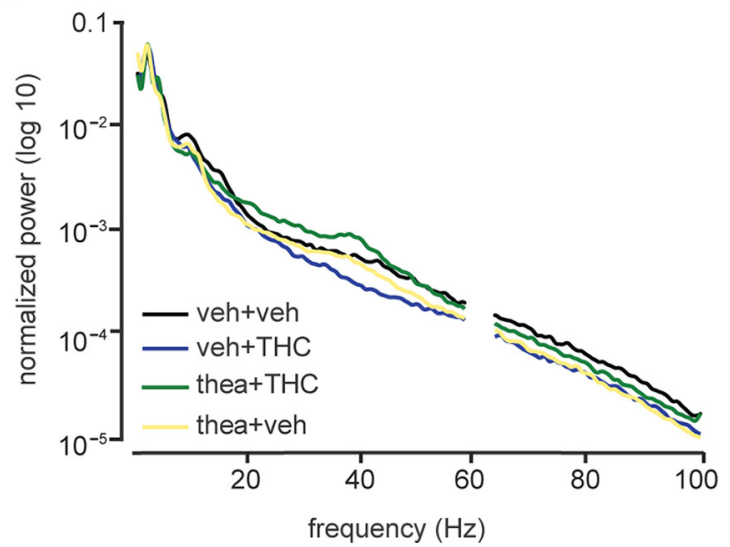

C

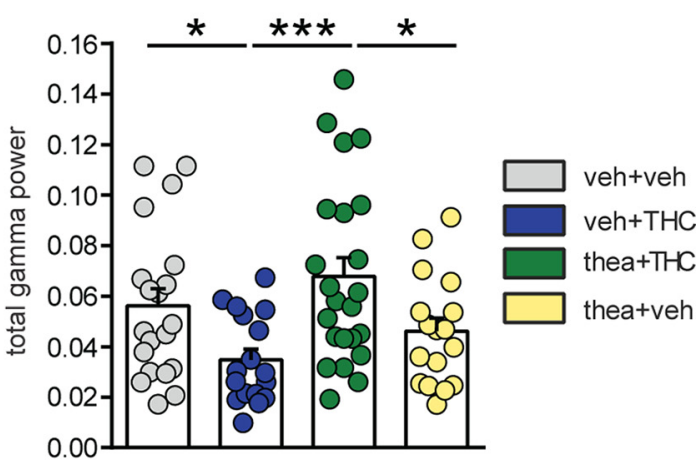

Figure 4. Effect of $\mathrm{L}$-theanine on THC-induced abnormalities in spontaneous cortical gamma oscillations $(30-80 \mathrm{~Hz})$. $\boldsymbol{A}$, Representative spectrogram of a 5 min recording during desynchronized state, characterized by small-amplitude fast oscillations (trace on the top). LFP power values between 59 and $61 \mathrm{~Hz}$ were excluded since they were reflecting the power line frequency. $\boldsymbol{B}$, Average normalized power spectra representing LFP during the desynchronized state in prefrontal cortex of the four experimental groups. The THC-treated group displayed a decrease in total gamma oscillations $(30-80 \mathrm{~Hz})$ during the desynchronized state. L-Theanine prevented THC-induced abnormalities in prefrontal LFP gamma oscillations. C, Group summary comparing LFP power following adolescent treatments. The THC-induced reduction in the total power of gamma oscillations in the desynchronized state was fully prevented by pre-exposure to L-theanine ( $n=20$ recording sites from 6 vehicle rats; $n=17$ recording sites from 3 THC rats; $n=23$ recording sites from 4 thea + THC rats; $n=17$ recording sites from 4 theanine rats; two-way ANCOVA, post hoc Fisher's LSD; $* p<0.05, * * * p<0.001)$.

al., 2001). As previously reported (Renard et al., 2017a), adolescent THC exposure significantly impairs sensorimotor gating in adulthood. We found that L-theanine fully prevented this THCinduced sensory-gating deficit. While the precise mechanisms underlying THC-induced PPI deficits are not currently understood, normal gamma-oscillation activity in the sensory cortex is necessary for effective sensorimotor gating (Cheng et al., 2016). Given that L-theanine was able to prevent the development of PFC-related gamma-oscillation disturbances, this effect may relate to the preventative effects of theanine on THC-induced cognitive filtering impairments. Moreover, both the Akt and GSK-3 PFC signaling pathways are crucial for regulating normal sensorimotor gating behaviors. For example, Kapfhamer et al. (2010) reported that GSK-3 signaling was critical for modulating M-type potassium channel function on PFC pyramidal neuron activity during PPI behavioral processing, such that the inhibition of GSK-3 signaling led to substantial PPI deficits. In addition, Chen and Lai (2011) reported that genetic knockdown of Akt1 caused profound impairments in PPI behaviors, which were reversed with GSK-3 inhibition, further underscoring the importance of these pathways in sensorimotor gating. We observed profound reductions in phosphorylated levels of PFC GSK-3 $\alpha / \beta$ and Akt-Thr308 levels following adolescent THC exposure, concomitant with dysregulated PFC neuronal activity states. While future studies are required to examine the causal mechanisms associated with these molecular adaptations, the ability of L-theanine to prevent these THC-induced molecular and neuronal phenotypes may underlie the normalization of long-term sensorimotor gating impairments. Interestingly, a previous report demonstrated that a single administration of 
A
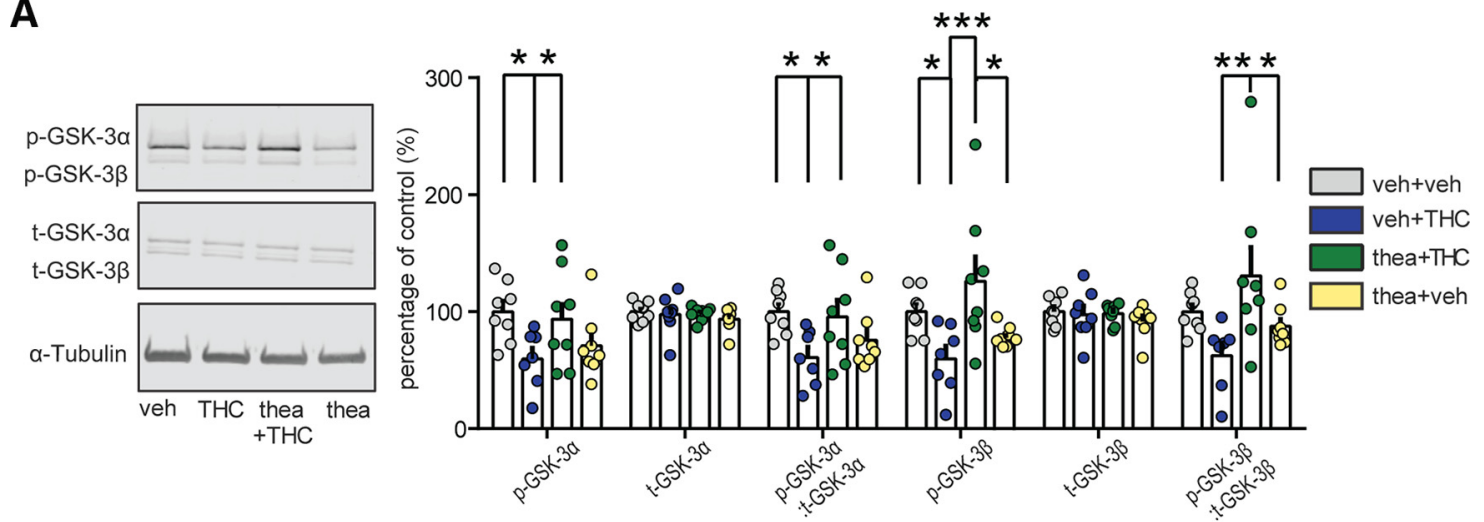

B

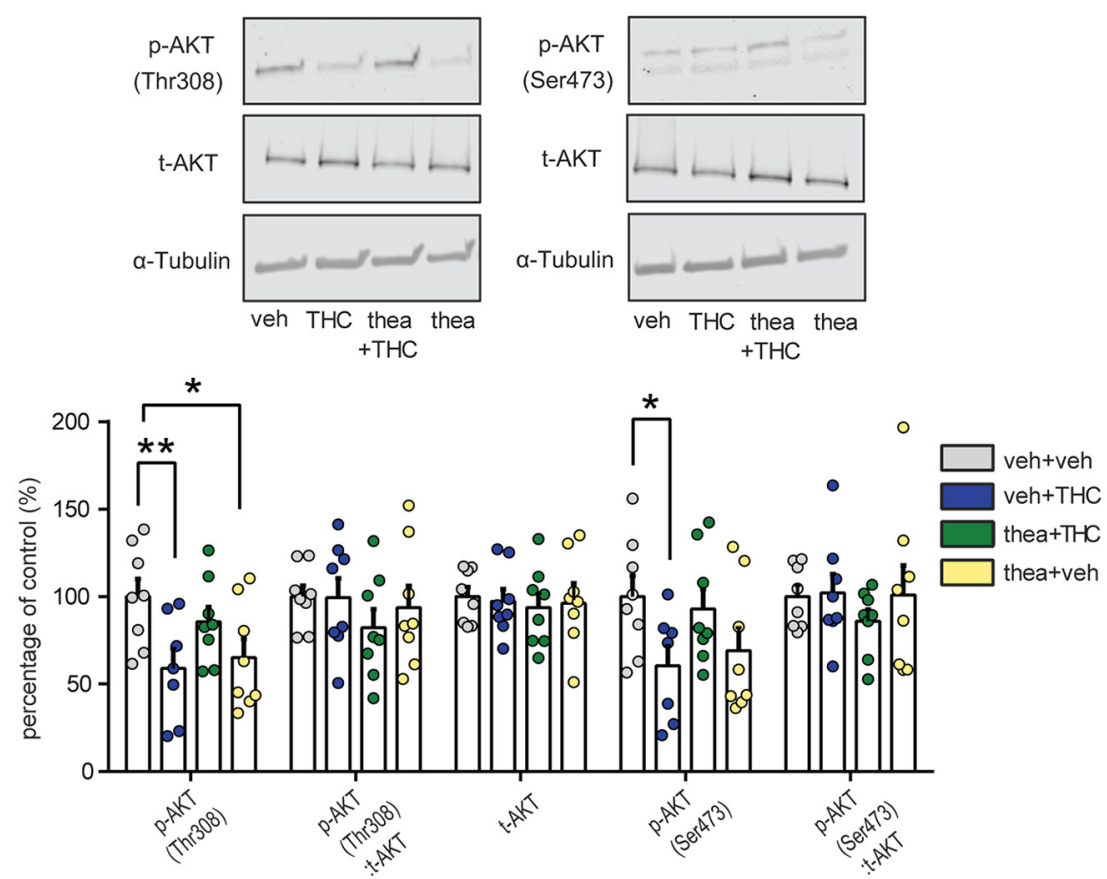

Figure 5. Effects of L-theanine on the expression of THC-induced schizophrenia-like abnormalities in molecular biomarkers. $A$, Representative Western blots for phosphorylated and total GSK-3 $\alpha$ and $\beta$ expression (left) in the PFC. Densitometry analysis revealed that L-theanine reverts THC-induced decreases in both GSK-3 isoforms, $\alpha$ and $\beta$, and in the ratio of phosphorylated to total GSK-3 $\alpha$ and GSK-3 $\beta$ (vehicle, $n=8$; THC, $n=7$; thea + THC, $n=8$; theanine, $n=8$; two-way ANOVA, post hoc Fisher's LSD; $* p<0.05, * * p<0.01, * * * p<0.001$ ). No differences were detected in total GSK-3 $\alpha$ and GSK-3 $\beta$ ( $n=8$ for each group). $\boldsymbol{B}$, Representative Western blots for phosphorylated and total AKT expression in PFC (top). L-Theanine coadministration normalized reductions in phosphorylated AKT-Thr308 and in the ratio of phosphorylated to total AKT-Thr308 induced by THC exposure during adolescence (vehicle, $n=8 ;$ THC, $n=7$; thea + THC, $n=8$; theanine, $n=8$; two-way ANOVA, post hoc Fisher's LSD; $* p<0.05, * * p<0.01$ ). No significant changes in phosphorylated AKT-Ser473 and in the ratio of phosphorylated to total AKT-Ser473 as well as in total AKT were detected ( $n=8$ for each group).

L-theanine was able to ameliorate PPI impairments induced by MK-801 (Wakabayashi et al., 2012), suggesting that the potential benefits of L-theanine on THC-induced cognitive impairments may extend beyond cannabinoid-related signaling mechanisms.

Cognitive impairments associated with schizophrenia have been correlated with abnormal cortical oscillation patterns, which are crucial for the normal coordination of excitatory versus inhibitory neural elements within many neural circuits, including the mesocorticolimbic system (Uhlhaas and Singer, 2010). Clinical and preclinical studies have described dysregulation of cortical gamma-band frequencies linked to schizophrenia-related cognitive abnormalities (Uhlhaas and Singer, 2010; Williams and Boksa, 2010; Raver et al., 2013). In the present study, we observed a persistent dysregulation in gamma band (frequency powers between 30 and $80 \mathrm{~Hz}$ ) in desynchronized states, in the PFC of adolescent THC-exposed rats. Interestingly, L-theanine administration was able to prevent these THC- induced oscillatory disturbances. While we are not aware of any previous studies examining the role of $\mathrm{L}$-theanine in cortical gamma-oscillatory regulation, the effects of $\mathrm{L}$-theanine may relate to the normalization of THC-induced pyramidal neuron bursting disturbances and concomitant normalization of GABAergic/ glutamatergic signaling disturbances following adolescent THC exposure. For example, acute activation of PFC CB1R transmission has been reported to cause profound disturbances in associative emotional memory formation by causing abnormal associative bursting activity states in PFC neuronal populations (Laviolette and Grace, 2006; Tan et al., 2010), likely because of a loss of normal GABAergic inhibition of PFC pyramidal output neurons. Moreover, adolescent THC exposure causes long-term loss of GABAergic molecular markers directly in the PFC and a concomitant increase in PFC pyramidal neuron bursting levels (Renard et al., 2017b). Other neurodevelopmental animal models of schizophrenia have shown that reduced cortical density of 
parvalbumin-positive interneurons was associated with a reduction in gamma-band activity patterns (Lodge et al., 2009). Given our findings that L-theanine prevented THC-induced gammaband disturbances as well as blocked the development of longterm hyperbursting levels in the PFC, one possibility is that Ltheanine prevents THC-induced disruptions in inhibitory/excitatory balance within the PFC by its ability to prevent subcortical overdrive of DA signals to the PFC.

Such a mechanism is supported by the present molecular findings in the PFC Akt-GSK signaling pathway. For example, both phosphorylated Akt and GSK-3 are strongly reduced in postmortem cortical schizophrenia tissue (Emamian, 2012) as well as in the rat PFC following chronic adolescent THC exposure (Renard et al., 2017a). Changes in cortical GSK-3 $\alpha / \beta$ and AKT, particularly in Thr308, are associated with hyperdopaminergic states, as observed following adolescent THC exposure (Renard et al., 2017a,b). Moreover, PFC GSK-3 $\alpha / \beta$ and Akt is reduced following chronic $\mathrm{DA} \mathrm{D}_{2}$ receptor activation (Sutton and Rushlow, 2012), which is consistent with the subcortical overdrive of VTA DAergic signals observed in the present study and the ability of L-theanine to prevent this hyperactive DA signal. Importantly, adolescent THC exposure induced a profound reduction in $\mathrm{p}-\mathrm{GSK}-3 \alpha / \beta$ expression and in the ratio of both ( $\mathrm{p}$ GSK- $3 \alpha$ /total p-GSK- $3 \alpha$ and p-GSK- $3 \beta$ /total GSK- $3 \beta$ ) as well as in p-Akt and its ratio, exclusively at Thr308. The selective effects of L-theanine at Akt Thr308 is of particular translational significance given that genetic biomarkers associated with AktThr308 are directly linked to an increased risk of cannabisrelated psychosis vulnerability (Di Forti et al., 2012). Finally, Ltheanine protects against DA-dependent neurotoxic effects in vitro. For example, L-theanine blocked excess DA-related toxicity in cultured mesencephalic neurons by increasing glutathione levels in surrounding astrocytes (Takeshima et al., 2016). While the present studies were performed in vivo, such evidence may suggest that L-theanine might similarly protect against DAergic toxicity in frontal cortical regions through similar modulation of astrocytic populations. Future studies are required to further explore these possibilities.

In conclusion, we report a novel neuroprotective role for Ltheanine in mitigating the neuropsychiatric side effects of chronic adolescent THC exposure. The range of neuroprotective effects induced by L-theanine were remarkable not only for their persistence beyond the adolescent THC exposure period, but for the comprehensive nature of its protective effects. These benefits extended beyond the prevention of THC-induced affective and cognitive disturbances and included the prevention of long-term molecular and neuronal adaptations within the PFC and a concomitant normalization of subcortical DAergic abnormalities.

\section{References}

Alimohamad H, Rajakumar N, Seah YH, Rushlow W (2005) Antipsychotics alter the protein expression levels of $\beta$-catenin and GSK-3 in the rat medial prefrontal cortex and striatum. Biol Psychiatry 57:533-542.

Andréasson S, Allebeck P, Engström A, Rydberg U (1987) Cannabis and schizophrenia. A longitudinal study of Swedish conscripts. Lancet 2:1483-1486.

Arseneault L, Cannon M, Poulton R, Murray R, Caspi A, Moffitt TE, Arseneault L, Cannon M, Murray R, Poulton R (2002) Cannabis use in adolescence and risk for adult psychosis: longitudinal prospective study. BMJ 325:1212-1213.

Bambico FR, Nguyen N, Katz N, Gobbi G (2010) Chronic exposure to cannabinoids during adolescence but not during adulthood impairs emotional behaviour and monoaminergic neurotransmission. Neurobiol Dis 37:641-655.
Braff DL, Geyer MA (1990) Sensorimotor gating and schizophrenia. human and animal model studies. Arch Gen Psychiatry 47:181-188.

Chen S, Wang Z, Ma Y, Zhang W, Lu J, Liang Y, Zheng X (2018) Neuroprotective effects and mechanisms of tea bioactive components in neurodegenerative diseases. Molecules 23:512.

Chen YW, Lai WS (2011) Behavioral phenotyping of v-akt murine thymoma viral oncogene homolog 1-deficient mice reveals a sex-specific prepulse inhibition deficit in females that can be partially alleviated by glycogen synthase kinase- 3 inhibitors but not by antipsychotics. Neuroscience 174:178-189.

Cheng CH, Chan PYS, Niddam DM, Tsai SY, Hsu SC, Liu CY (2016) Sensory gating, inhibition control and gamma oscillations in the human somatosensory cortex. Sci Rep 6:20437.

Cuccurazzu B, Zamberletti E, Nazzaro C, Prini P, Trusel M, Grilli M, Parolaro D, Tonini R, Rubino T (2018) Adult cellular neuroadaptations induced by adolescent THC exposure in female rats are rescued by enhancing anandamide signaling. Int J Neuropsychopharmacol 21:10141024.

Di Forti M, Iyegbe C, Sallis H, Kolliakou A, Falcone MA, Paparelli A, Sirianni M, La Cascia C, Stilo SA, Marques TR, Handley R, Mondelli V, Dazzan P, Pariante C, David AS, Morgan C, Powell J, Murray RM (2012) Confirmation that the AKT1 (rs2494732) genotype influences the risk of psychosis in cannabis users. Biol Psychiatry 72:811-816.

Draycott B, Loureiro M, Ahmad T, Tan H, Zunder J, Laviolette SR (2014) Cannabinoid transmission in the prefrontal cortex bi-phasically controls emotional memory formation via functional interactions with the ventral tegmental area. J Neurosci 34:13096-13109.

Emamian ES (2012) AKT/GSK3 signaling pathway and schizophrenia. Front Mol Neurosci 5:33.

Ferdinand RF, Sondeijker F, Van Der Ende J, Selten J, Huizink A, Verhulst FC (2005) Cannabis use predicts future psychotic symptoms, and vice versa. Addiction 100:612-618.

Fergusson DM, Horwood LJ, Swain-Campbell NR (2003) Cannabis dependence and psychotic symptoms in young people. Psychol Med 33:15-21.

Gururajan A, Van Den Buuse M (2014) Is the mTOR-signalling cascade disrupted in Schizophrenia? J Neurochem 129:377-387.

Herkenham M, Lynn AB, Little MD, Johnson MR, Melvin LS, De Costa BR, Rice KC (1990) Cannabinoid receptor localization in brain. Proc Natl Acad Sci U S A 87:1932-1936.

Kakuda T (2011) Neuroprotective effects of theanine and its preventive effects on cognitive dysfunction. Pharmacol Res 64:162-168.

Kakuda T, Hinoi E, Abe A, Nozawa A, Ogura M, Yoneda Y (2008) Theanine, an ingredient of green tea, inhibits $[3 \mathrm{H}]$ glutamine transport in neurons and astroglia in rat brain. J Neurosci Res 86:1846-1856.

Kakuda TK, Ozawa AN, Ugimoto AS, Niino H (2002) Inhibition by theanine of binding of [3H]AMPA, [3H]kainate, and [3H]MDL 105,519 to glutamate receptors. Biosci Biotechnol Biochem 66:2683-2686.

Kambeitz J, Abi-Dargham A, Kapur S, Howes OD (2014) Alterations in cortical and extrastriatal subcortical dopamine function in schizophrenia: systematic review and meta-analysis of imaging studies. Br J Psychiatry 204:420-429.

Kapfhamer D, Berger KH, Hopf FW, Seif T, Kharazia V, Bonci A, Heberlein U (2010) Protein phosphatase 2A and glycogen synthase kinase 3 signaling modulate prepulse inhibition of the acoustic startle response by altering cortical M-type potassium channel activity. J Neurosci 30:8830-8840.

Krebs M-O, Kebir O, Jay TM (2019) Exposure to cannabinoids can lead to persistent cognitive and psychiatric disorders. Eur J Pain 23:1225-1233.

Lardner AL (2014) Neurobiological effects of the green tea constituent theanine and its potential role in the treatment of psychiatric and neurodegenerative disorders. Nutr Neurosci 17:145-155.

Laviolette SR, Grace AA (2006) Cannabinoids potentiate emotional learning plasticity in neurons of the medial prefrontal cortex through basolateral amygdala inputs. J Neurosci 26:6458-6468.

Lee J, Nuechterlein KH, Knowlton BJ, Bearden CE, Cannon TD, Fiske AP, Ghermezi L, Hayata JN, Hellemann GS, Horan WP, Kee K, Kern RS, Subotnik KL, Sugar CA, Ventura J, Yee CM, Green MF (2018) Episodic memory for dynamic social interaction across phase of illness in schizophrenia. Schizophr Bull 44:620-630.

Llorente-Berzal A, Puighermanal E, Burokas A, Ozaita A, Maldonado R, Marco EM, Viveros M-P (2013) Sex-dependent psychoneuroendocrine effects of THC and MDMA in an animal model of adolescent drug consumption. PLoS One 8:e78386. 
Lodge DJ, Behrens MM, Grace AA (2009) Neurobiology of disease a loss of parvalbumin-containing interneurons is associated with diminished oscillatory activity in an animal model of schizophrenia. J Neurosci 29:2344-2354.

Malone DT, Hill MN, Rubino T (2010) Adolescent cannabis use and psychosis: epidemiology and neurodevelopmental models. Br J Pharmacol 160:511-522.

Murphy M, Mills S, Winstone J, Leishman E, Wager-Miller J, Bradshaw H, Mackie K (2017) Chronic adolescent $\Delta 9$-tetrahydrocannabinol treatment of male mice leads to long-term cognitive and behavioral dysfunction, which are prevented by concurrent cannabidiol treatment. Cannabis Cannabinoid Res 2:235-246.

Murray RM, Englund A, Abi-dargham A, Lewis DA, Di Forti M, Davies C, Sherif M, Mcguire P, Souza DCD (2017) Cannabis-associated psychosis: neural substrate and clinical impact. Neuropharmacology 124:89-104.

Nathan PJ, Lu K, Gray M, Oliver C (2006) The neuropharmacology of L-theanine (N-ethyl-L-glutamine): a possible neuroprotective and cognitive enhancing agent. J Herb Pharmacother 6:21-30.

O'Shea M, McGregor IS, Mallet PE (2006) Repeated cannabinoid exposure during perinatal, adolescent or early adult ages produces similar longlasting deficits in object recognition and reduced social interaction in rats. J Psychopharmacol 20:611-621.

Ota M, Wakabayashi C, Sato N, Hori H, Hattori K, Teraishi T, Ozawa H, Okubo T, Kunugi H (2015) Effect of L-theanine on glutamatergic function in patients with schizophrenia. Acta Neuropsychiatr 27:291-296.

Park SK, Jung IC, Lee WK, Lee YS, Park HK, Go HJ, Kim K, Lim NK, Hong JT, Ly SY, Rho SS (2011) A combination of green tea extract and L-theanine improves memory and attention in subjects with mild cognitive impairment: a double-blind placebo-controlled study. J Med Food 14:334-343.

Paxinos G, Watson C (2007) The rat brain in stereotaxic coordinates. San Diego: Academic.

Quinn HR, Matsumoto I, Callaghan PD, Long LE, Arnold JC, Gunasekaran N, Thompson MR, Dawson B, Mallet PE, Kashem MA, MatsudaMatsumoto H, Iwazaki T, McGregor IS (2008) Adolescent rats find repeated $\Delta^{9}$-THC less aversive than adult rats but display greater residual cognitive deficits and changes in hippocampal protein expression following exposure. Neuropsychopharmacology 33:1113-1126.

Raver SM, Haughwout SP, Keller A (2013) Adolescent cannabinoid exposure permanently suppresses cortical oscillations in adult mice. Neuropsychopharmacology 38:2338-2347.

Renard J, Krebs M, Jay TM, Le Pen G (2013) Long-term cognitive impairments induced by chronic cannabinoid exposure during adolescence in rats: a strain comparison. Psychopharmacology (Berl) 225:781-790.

Renard J, Rosen LG, Loureiro M, De Oliveira C, Schmid S, Rushlow WJ, Laviolette SR (2017a) Adolescent cannabinoid exposure induces a persistent sub-cortical hyper-dopaminergic state and associated molecular adaptations in the prefrontal cortex. Cereb Cortex 27:1297-1310.

Renard J, Szkudlarek HJ, Kramar CP, Jobson CEL, Moura K, Rushlow WJ, Laviolette SR (2017b) Adolescent THC exposure causes enduring prefrontal cortical disruption of GABAergic inhibition and dysregulation of sub-cortical dopamine function. Sci Rep 7:11420.

Renard J, Rushlow WJ, Laviolette SR (2018) Effects of adolescent THC exposure on the prefrontal GABAergic system: implications for schizophrenia-related psychopathology. Front Psychiatry 9:1-13.

Ritsner MS, Miodownik C, Ratner Y, Shleifer T, Mar M, Pintov L, Lerner V (2011) L-theanine relieves positive, activation, and anxiety symptoms in patients with schizophrenia and schizoaffective disorder: an 8-week, randomized, double-blind, placebo-controlled, 2-center study. J Clin Psychiatry 72:34-42.

Rubino T, Parolaro D (2016) The impact of exposure to cannabinoids in adolescence: insights from animal models. Biol Psychiatry 79:578-585.

Rubino T, Vigano' D, Realini N, Guidali C, Braida D, Capurro V, Castiglioni C, Cherubino F, Romualdi P, Candeletti S, Sala M, Parolaro D (2008) Chronic delta-9-tetrahydrocannabinol during adolescence provokes sexdependent changes in the emotional profile in adult rats: behavioral and biochemical correlates. Neuropsychopharmacology 33:2760-2771.

Rubino T, Realini N, Braida D, Guidi S, Capurro V, Viganò D, Guidali C, Pinter M, Sala M, Bartesaghi R, Parolaro D (2009) Changes in hippocampal morphology and neuroplasticity induced by adolescent THC treatment are associated with cognitive impairment in adulthood. Hippocampus 19:763-772.

Schneider M, Koch M (2003) Chronic pubertal, but not adult chronic cannabinoid treatment impairs sensorimotor gating, recognition memory, and the performance in a progressive ratio task in adult rats. Neuropsychopharmacology 28:1760-1769.

Segal-Gavish H, Gazit N, Barhum Y, Ben-Zur T, Taler M, Hornfeld SH, GilAd I, Weizman A, Slutsky I, Niwa M, Kamiya A, Sawa A, Offen D, Barzilay R (2017) BDNF overexpression prevents cognitive deficit elicited by adolescent cannabis exposure and host susceptibility interaction. Hum Mol Genet 26:2462-2471.

Shen M, Yang Y, Wu Y, Zhang B, Wu H, Wang L, Tang H, Chen J (2019) L-theanine ameliorate depressive-like behavior in a chronic unpredictable mild stress rat model via modulating the monoamine levels in limbic-corticalstriatal-pallidal-thalamic-circuit related brain regions. Phytother Res 33:412-421.

Stefanis NC, Delespaul P, Henquet C, Bakoula C, Stefanis CN, Van Os J (2004) Early adolescent cannabis exposure and positive and negative dimensions of psychosis. Addiction 99:1333-1341.

Sutton LP, Rushlow WJ (2012) The dopamine D2 receptor regulates Akt and GSK-3 via Dvl-3. Int J Neuropsychopharmacol 15:965-979.

Swerdlow NR, Geyer MA, Braff DL (2001) Neural circuit regulation of prepulse inhibition of startle in the rat: current knowledge and future challenges. Psychopharmacology (Berl) 156:194-215.

Takeshima M, Miyazaki I, Murakami S, Kita T, Asanuma M (2016) LTheanine protects against excess dopamine-induced neurotoxicity in the presence of astrocytes. J Clin Biochem Nutr 59:93-99.

Tamano H, Fukura K, Suzuki M, Sakamoto K, Yokogoshi H, Takeda A (2013) Preventive effect of theanine intake on stress-induced impairments of hippocampal long-term potentiation and recognition memory. Brain Res Bull 95:1-6.

Tan H, Lauzon NM, Bishop SF, Bechard MA, Laviolette SR (2010) Integrated cannabinoid CB1 receptor transmission within the amygdala-prefrontal cortical pathway modulates neuronal plasticity and emotional memory encoding. Cereb Cortex 20:1486-1496.

Tan H, Lauzon NM, Bishop SF, Chi N, Bechard M, Laviolette SR (2011) Cannabinoid transmission in the basolateral amygdala modulates fear memory formation via functional inputs to the prelimbic cortex. J Neurosci 31:5300-5312.

Uhlhaas PJ, Singer W (2010) Abnormal neural oscillations and synchrony in schizophrenia. Nat Rev Neurosci 11:100-113.

Wakabayashi C, Numakawa T, Ninomiya M, Chiba S, Kunugi H (2012) Behavioral and molecular evidence for psychotropic effects in L-theanine. Psychopharmacology (Berl) 219:1099-1109.

Wegener N, Koch M (2009) Behavioural disturbances and altered Fos protein expression in adult rats after chronic pubertal cannabinoid treatment. Brain Res 1253:81-91.

Weiser M, Knobler HY, Noy S, Kaplan Z (2002) Clinical characteristics of adolescents later hospitalized for schizophrenia. Am J Med Genet 114:949-955

Williams S, Boksa P (2010) Gamma oscillations and schizophrenia. J Psychiatry Neurosci 35:75-77.

Yin C, Gou L, Liu Y, Yin X, Zhang L, Jia G, Zhuang X (2011) Antidepressant-like effects of 1-theanine in the forced swim and tail suspension tests in mice. Phytother Res 25:1636-1639.

Zamberletti E, Beggiato S, Steardo L, Prini P, Antonelli T, Ferraro L, Rubino T, Parolaro D (2014) Neurobiology of Disease Alterations of prefrontal cortex GABAergic transmission in the complex psychotic-like phenotype induced by adolescent delta-9-tetrahydrocannabinol exposure in rats. Neurobiol Dis 63:35-47.

Zammit S, Allebeck P, Andreasson S, Lundberg I, Lewis G (2002) Self reported cannabis use as a risk factor for historical cohort study. BMJ 325:1199.

Zukhurova M, Prosvirnina M, Daineko A, Simanenkova A, Petrishchev N, Sonin D, Galagudza M, Shamtsyan M, Juneja L, Vlasov T (2013) L-theanine administration results in neuroprotection and prevents glutamate receptor agonist-mediated injury in the rat model of cerebral ischemiareperfusion. Phytother Res 27:1282-1287. 